\title{
Stability Analysis of Herringbone-Grooved Aerodynamic Journal Bearings for Ultra High-Speed Rotations
}

\author{
Norifumi Miyanaga and Jun Tomioka
}

\begin{abstract}
Herringbone-grooved aerodynamic journal bearings are suitable to support a rotating shaft with an ultra high-speed rotation. The aim of this work is to investigate the effect of herringbone-groove geometries on stability characteristics of the bearings, considering the centrifugal growth of the shaft. For this purpose, a parametric study of groove geometry is presented. The pressure in a lubricant air film is governed by the compressive lubrication equation based on the Narrow Groove Theory. The dynamic coefficients of the bearing system are calculated using the linear perturbation method. The threshold speed of whirl instability was determined by applying the Routh-Hurwitz criterion to the characteristic equation of the bearing system, and then the stability chart were drawn. The validity of this analysis was proved by the comparison of the stability chart with the journal behaviors obtained from the nonlinear transient analysis. The results obtained from the perturbation analysis and the nonlinear transient analysis was in good agreement. These analysis shows that the effect of the centrifugal growth of the shaft on the threshold speed of whirl instability was significant in high rotational speed. By considering the effect, the proper herringbone-groove geometries for ultra high-speed operations were obtained in this paper.
\end{abstract}

Index Terms-Herringbone bearings, aerodynamic bearings, ultra high-speed rotations, stability analysis.

\section{INTRODUCTION}

For compact rotating machines, it is important to achieve a stable ultra high-speed shaft rotation for superior performance. Air-lubricated journal bearings are suitable to be used in such small rotating machines due to their low frictional loss, compared with oil bearings and rolling bearings. However, it is absolutely difficult to apply them to such machineries since they induce the self-excited vibration called whirl instability in high-speed operating conditions.

Aerostatic bearings have been studied for high-speed rotational devices [1]-[3]. Hikichi et al. [4] developed a hydroinertia air bearings for over a million rpm. This bearing is a special type of aerostatic bearings, in which the inertia effect of air flow in a bearing clearance is predominant to the viscous effect. Then, Tanaka et al. [5], [6] applied this bearings to a palmtop gas turbine. As mentioned in these references, aerostatic bearings can support a rotating shaft with a rotational speed over a million $\mathrm{rpm}$. However, in

Manuscript received April 15, 2015; revised July 23, 2015.

Norifumi Miyanaga is with Kanto-Gakuin University, 1-50-1 Mutsuura-Higashi, Kanazawa-Ku, Yokohama, Japan (e-mail: miyanaga@kanto-gakuin.ac.jp).

Jun Tomioka is with Waseda University, 3-4-1 Okubo, Shinjuku-Ku, Tokyo, Japan (e-mail: tomioka@waseda.jp). aerostatic bearings, a lubricating air film is provided from an external source. Thus, they are not proper for the practical uses in the compact machines. On the other hand, in aerodynamic bearings, the generation of a lubricating air film relies on the geometry and motion of the bearings. Among aerodynamic bearings, foil bearings have been extensively studied as the strong candidates for ultrahigh speed operations [7]-[10]. Typical foil bearings have high structural damping by dry friction between the top foil and the bump foil. However, because of the wear between the foils, they need routine maintenance. In addition, it seems that the optimal design of the bearing configurations is not easy because of their complex structures.

Currently, herringbone-grooved bearings have been used in miniature rotating machines such as information devices [11]-[15]. They have herringbone-shape grooves on their bearing surface, and can generate higher support stiffness by pumping a lubricating fluid inward. For that reasons, they can be expected to operate up to the ultra high-speed without the unstable phenomena. Therefore, in the previous paper, the authors [16] developed an aerodynamic bearing system employing herringbone-grooved aerodynamic journal bearings and tested its bearing performance. In this system, a rotating shaft with $6.0 \mathrm{~mm}$ diameter and $30.75 \mathrm{~mm}$ length is supported by the herringbone-grooved aerodynamic journal bearings at both ends. The shaft is driven by an air turbine located at its one end. According to the tests, the system can be operated up to a half million rpm, and the threshold speed of whirl instability of the herringbone-grooved bearings were extremely higher than that of the plane bearings. Yoshimoto $e t$ al. [17] also reported that herringbone-grooved bearings have the ability to be used in their ultra micro gas turbine. Ise $e t$ al. [18] developed a split-type herringbone-grooved journal bearing and demonstrated its performance. In this demonstration, the bearing induced the whirl instability at very low rotational speed.

The performance of herringbone-grooved aerodynamic journal bearings, including the threshold speed of whirl instability, is strongly influenced by the groove geometries. However, the effects of the groove geometries on the stability of the herringbone-grooved bearings for ultra high-speed rotations are not fully discussed yet. The purpose of this present work is to establish the effects of the grooves on the stability of the herringbone-grooved bearings, and to clarify the proper groove geometry for more ultra high-speed operations. For this purpose, a parametric study of groove geometry is presented by the linear perturbation stability analysis. A nonlinear transient stability analysis is also presented. These analyses incorporate the effect of the centrifugal growth of the rotating shaft. 


\section{NOMENCLATURE}

The nomenclatures used in this paper are as follows. Variables with $\wedge$ means dimensional quantities and those without $\wedge$ means non-dimensional.

$\hat{C}$ : bearing radial clearance

$\hat{e}$ : eccentricity

$h:$ fluid-film thickness $=\hat{h} / \hat{C}$

$H$ : groove depth ratio $=(\hat{\delta}+\hat{C}) / \hat{C}$

$\hat{L}$ : bearing length

$\hat{m}$ : mass of shaft

$p:$ fluid-film pressure $=\hat{p} / \hat{p}_{a}$

$\hat{p}_{a}:$ atmospheric pressure

$\hat{R}$ : shaft radius

$W:$ fluid-film force $=\hat{W} /\left(2 \hat{p}_{a} \hat{R} \hat{L}\right)$

$\alpha:$ groove width ratio $=\hat{\alpha}_{g} /\left(\hat{\alpha}_{r}+\hat{\alpha}_{g}\right)$

$\hat{\alpha}_{r}$ : width of ridge part in a ridge-groove pair

$\hat{\alpha}_{g}$ : width of groove part in a ridge-groove pair

$\hat{\beta}$ : groove angle

$\hat{\delta}$ : groove depth

$\varepsilon:$ eccentricity ratio $=\hat{e} / \hat{C}$

$\lambda$ : bearing length to diameter ratio $=\hat{L} / 2 \hat{R}$

$\Lambda:$ dimensionless rotational speed

$\hat{\mu}$ : viscosity of fluid-film

$v:$ whirl frequency ratio $=\hat{\omega}_{p} / \hat{\omega}$

$\tau:$ time $=\hat{\omega} \hat{t}$

$\hat{\omega}$ : angular velocity of shaft rotation

$\hat{\omega}_{p}$ : angular velocity of whirl

$\Omega:$ shaft mass $=\hat{m} \hat{p}_{a} \hat{C}^{5} /\left(\hat{\mu}^{2} L \hat{R}^{5}\right)$

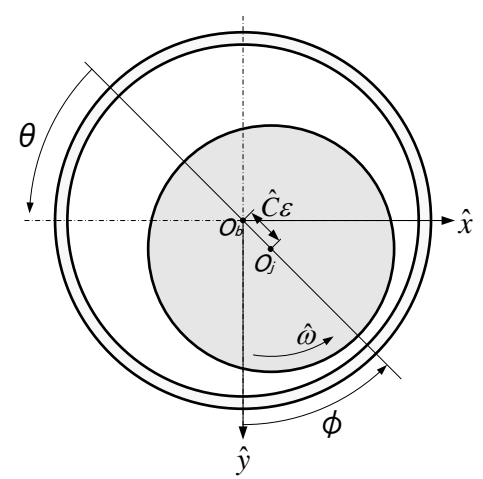

Fig. 1. Journal bearing.

\section{THEORY}

\section{A. Lubrication Equation}

The coordinate of the herringbone-grooved bearing analyzed here is shown in Fig. 1 - Fig. 2. The bearing sleeve has the grooves on its inner surface as shown in Fig. 2. The pressure in a lubricant air film is governed by the compressive lubrication equation based on the narrow groove theory [19]. This theory provides reasonable solutions to bearings with large number of herringbone grooves. Assuming the perfect gas and the isothermal conditions, the lubrication equation is

$$
\begin{aligned}
& \frac{\partial}{\partial \theta}\left[\left(\frac{E_{1}}{E_{0}}\right) \frac{\partial p^{2}}{\partial \theta}+\frac{1}{\lambda}\left(\frac{E_{2}}{E_{0}}\right) \frac{\partial p^{2}}{\partial z}-2 \Lambda\left(\frac{E_{3}}{E_{0}}\right) p+2 \Lambda E_{4} p\right] \\
& +\frac{1}{\lambda} \frac{\partial}{\partial z}\left[\left(\frac{E_{5}}{E_{0}}\right) \frac{\partial p^{2}}{\partial \theta}+\frac{1}{\lambda}\left(\frac{E_{6}}{E_{0}}\right) \frac{\partial p^{2}}{\partial z}-2 \Lambda\left(\frac{E_{7}}{E_{0}}\right) p\right] \\
& -2 \Lambda\left[2\left(\frac{\partial}{\partial \tau}-\frac{\partial \phi}{\partial \tau} \frac{\partial}{\partial \theta}\right)+\frac{\partial}{\partial \theta}\right] E_{8} p=0
\end{aligned}
$$

where the nomenclatures of $E_{0}-E_{8}$ are the functions of the groove geometry $(\alpha, \beta, H)$ and film thickness $(h)$, shown as follows.

$$
\begin{aligned}
& E_{0}=\alpha h_{r}{ }^{3}+(1-\alpha) h_{g}{ }^{3} \\
& E_{1}=h_{r}{ }^{3} h_{g}{ }^{3}+\alpha(1-\alpha)\left(h_{g}{ }^{3}-h_{r}{ }^{3}\right) \cos ^{2} \beta \\
& E_{2}=\alpha(1-\alpha)\left(h_{g}{ }^{3}-h_{r}{ }^{3}\right)^{2} \cos \beta \sin \beta \\
& E_{3}=\alpha\left(h_{g}-h_{r}\right) h_{r}{ }^{3}+\alpha(1-\alpha)\left(h_{g}-h_{r}\right)\left(h_{g}{ }^{3}-h_{r}{ }^{3}\right) \cos ^{2} \beta \\
& E_{4}=\alpha\left(h_{g}-h_{r}\right) \\
& E_{5}=\alpha(1-\alpha)\left(h_{g}{ }^{3}-h_{r}{ }^{3}\right)^{2} \sin \beta \cos \beta \\
& E_{6}=h_{r}{ }^{3} h_{g}{ }^{3}+\alpha(1-\alpha)\left(h_{g}{ }^{3}-h_{r}{ }^{3}\right) \sin ^{2} \beta \\
& E_{7}=\alpha(1-\alpha)\left(h_{g}-h_{r}\right)\left(h_{g}{ }^{3}-h_{r}{ }^{3}\right) \sin \beta \cos \beta \\
& E_{8}=\alpha h_{r}+(1-\alpha) h_{g}
\end{aligned}
$$
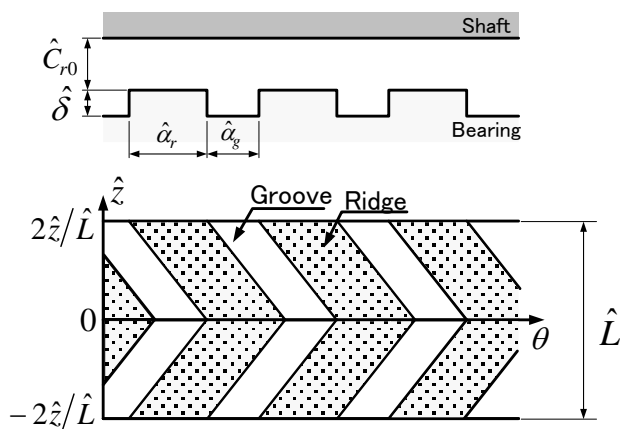

The film thickness is given for ridge and groove part, respectively, shown as follows.

$$
\begin{gathered}
h_{r}=1+\varepsilon \cos \theta-\psi \Lambda^{2} \text { (at ridge part) } \\
h_{g}=H+\varepsilon \cos \theta-\psi \Lambda^{2} \text { (at groove part) }
\end{gathered}
$$

where $\psi$ represents the expansion ratio of the rotating shaft and is expressed as

$$
\psi=\frac{\left(1-v_{p}\right) \hat{\rho} \hat{p}_{a}^{2} \hat{C}^{3}}{144 \hat{E} \hat{\mu}^{2} \hat{R}}
$$

In Eq.(5), $\hat{E}$ and $v_{p}$ are the Young's modulus (72GPa) and the poison ratio $(0.34)$ of the shaft, respectively. $\hat{\rho}$ is the 
density of the shaft $\left(2700 \mathrm{~kg} / \mathrm{m}^{3}\right)$.

The boundary conditions in the circumferential and axial directions are

$$
\begin{gathered}
p(\theta, \pm 1)=1 \\
p(\theta, z)=p(\theta+2 \pi, z) \\
\left.\frac{\partial p}{\partial \theta}\right|_{\theta}=\left.\frac{\partial p}{\partial \theta}\right|_{\theta+2 \pi} \\
{\left[\alpha\left(M_{z}\right)_{\text {groove }}+(1-\alpha)\left(M_{z}\right)_{\text {ridge }}\right]_{z=0}=0}
\end{gathered}
$$

\section{B. Steady-State Performance}

The components of the fluid-film forces in the radial and tangential directions at the static equilibrium position are obtained from Eqs.(10) and (11), respectively.

$$
\begin{gathered}
W_{\varepsilon}=-\frac{1}{4} \int_{-1}^{1} \int_{0}^{2 \pi}(p-1) \cos \theta d \theta d z \\
W_{\phi}=\frac{1}{4} \int_{-1}^{1} \int_{0}^{2 \pi}(p-1) \sin \theta d \theta d z
\end{gathered}
$$

The load carrying capacity and the attitude angle are obtained from Eqs.(12) and (13), respectively.

$$
\begin{gathered}
W=\sqrt{W_{\varepsilon}^{2}+W_{\phi}^{2}} \\
\phi=\tan ^{-1}\left(W_{\phi} / W_{\varepsilon}\right)
\end{gathered}
$$

\section{Equations of Motion}

Let us consider a simple bearing system, in which a perfectly balanced rigid shaft is supported on herringbone-grooved aerodynamic journal bearings. The equations of motion of the shaft in $\mathrm{x}$ - and $\mathrm{y}$-directions are

$$
\begin{gathered}
\left(\Omega \Lambda^{2} / 72\right) \ddot{X}=-W_{\varepsilon} \sin \phi+W_{\phi} \cos \phi \\
\left(\Omega \Lambda^{2} / 72\right) \ddot{Y}=-W_{\varepsilon} \cos \phi-W_{\phi} \sin \phi
\end{gathered}
$$

\section{Linear Perturbation Stability Analysis}

In this section, the threshold speed of whirl instability is obtained by the linear perturbation analysis. When the shaft has a small vibration around the static equilibrium position at the threshold speed of whirl instability, the fluid-film pressure is expanded as follows.

$$
\begin{aligned}
p=p_{0}+p_{\varepsilon o} \Delta \varepsilon+p_{\varepsilon 1} \Delta \dot{\varepsilon}+p_{\varepsilon 2} \Delta \ddot{\varepsilon}+\cdots \\
+p_{\phi o} \Delta \phi+p_{\phi 1} \Delta \dot{\phi}+p_{\phi 2} \Delta \ddot{\phi}+\cdots
\end{aligned}
$$

Assuming the small displacement of the shaft as follows,

$$
\Delta \varepsilon=\varepsilon_{1} e^{i\left(v \tau+\varphi_{\varepsilon}\right)}
$$

$$
\Delta \phi=\phi_{1} e^{i\left(v \tau+\varphi_{\phi}\right)}
$$

Then,

$$
\begin{aligned}
& \Delta \dot{\varepsilon}=i v \varepsilon_{1} e^{i\left(v \tau+\varphi_{\varepsilon}\right)}, \quad \Delta \ddot{\varepsilon}=-v^{2} \Delta \varepsilon, \cdots \\
& \Delta \dot{\phi}=i v \phi_{1} e^{i\left(v \tau+\varphi_{\phi}\right)}, \quad \Delta \ddot{\phi}=-v^{2} \Delta \phi, \cdots
\end{aligned}
$$

Substituting Eqs.(17) to (20) into Eq.(16), the pressure is expressed as Eq.(21).

$$
p=p_{0}+p_{1} \Delta \varepsilon+p_{2} \Delta \dot{\varepsilon}+p_{3} \Delta \phi+p_{4} \Delta \dot{\phi}
$$

where $p_{0}$ is the pressure at the static equilibrium position and $p_{1}-p_{4}$ are the perturbed pressures. Then, by substituting Eq.(21) into Eqs.(10) and (11), one can obtain the linearized fluid-film forces.

$$
\begin{aligned}
& W_{\varepsilon}=W_{\varepsilon 0}+k_{\varepsilon \varepsilon} \Delta \varepsilon+k_{\varepsilon \phi} \Delta \phi+b_{\varepsilon \varepsilon} \Delta \dot{\varepsilon}+b_{\varepsilon \phi} \Delta \dot{\phi} \\
& W_{\phi}=W_{\phi 0}+k_{\phi \varepsilon} \Delta \varepsilon+k_{\phi \phi} \Delta \phi+b_{\phi \varepsilon} \Delta \dot{\varepsilon}+b_{\phi \phi} \Delta \dot{\phi}
\end{aligned}
$$

where four stiffness coefficients $\left(k_{\varepsilon \varepsilon}, k_{\varphi_{\varepsilon}}, k_{\varepsilon \varphi}, k_{\varphi \varphi}\right)$ and four damping coefficients $\left(b_{\varepsilon \varepsilon}, b_{\varphi \varepsilon}, b_{\varepsilon \varphi}, b_{\varphi \varphi}\right)$ are calculated as follows.

$$
\begin{aligned}
& k_{\mathscr{C}}=-\frac{1}{4} \int_{-1}^{1} \int_{0}^{2 \pi} p_{1} \cos \theta d \theta d z \\
& k_{s \phi}=-\frac{1}{4} \int_{-1}^{1} \int_{0}^{2 \pi} p_{2} \cos \theta d \theta d z \\
& k_{\phi \varepsilon}=-\frac{1}{4} \int_{-1}^{1} \int_{0}^{2 \pi} p_{3} \cos \theta d \theta d z \\
& k_{\phi \phi}=-\frac{1}{4} \int_{-1}^{1} \int_{0}^{2 \pi} p_{4} \cos \theta d \theta d z \\
& b_{\check{ }}=\frac{1}{4} \int_{-1}^{1} \int_{0}^{2 \pi} p_{1} \sin \theta d \theta d z \\
& b_{s \phi}=\frac{1}{4} \int_{-1}^{1} \int_{0}^{2 \pi} p_{2} \sin \theta d \theta d z \\
& b_{\phi \varepsilon}=\frac{1}{4} \int_{-1}^{1} \int_{0}^{2 \pi} p_{3} \sin \theta d \theta d z \\
& b_{\phi \phi}=\frac{1}{4} \int_{-1}^{1} \int_{0}^{2 \pi} p_{4} \sin \theta d \theta d z
\end{aligned}
$$

Assuming the solutions of the equations of motion as $X=X_{1} e^{s \tau}, Y=Y_{1} e^{s \tau}$ and by considering the homogeneity of the equations, an fourth-order characteristic equation is derived.

$$
\sum_{n=0}^{4} a_{n} s^{n}=0
$$

where

$$
\begin{aligned}
& \alpha_{0}=A_{2} \\
& \alpha_{1}=A_{1} \\
& \alpha_{2}=A_{3}+\left(\Lambda^{2} \Omega / 72\right) A_{4} \\
& \alpha_{3}=\left(\Lambda^{2} \Omega / 72\right) A_{5} \\
& \alpha_{4}=\left(\Lambda^{2} \Omega / 72\right)^{2}
\end{aligned}
$$


and

$$
\begin{aligned}
& A_{1}=K_{1} B_{4}+K_{4} B_{1}-K_{2} B_{3}-K_{3} B_{2} \\
& A_{2}=K_{1} K_{4}-K_{2} K_{3} \\
& A_{3}=B_{1} B_{4}-B_{2} B_{3} \\
& A_{4}=K_{1}+K_{4} \\
& A_{5}=B_{1}+B_{4}
\end{aligned}
$$

By applying the Routh-Herwiz stability criterion to Eq.(25), one can obtain the relations about the threshold speed of whirl instability.

$$
\begin{gathered}
v^{2}=\left(A_{1}^{2}+A_{2} A_{5}^{2}-A_{1} A_{4} A_{5}\right) / A_{3} A_{5}^{2} \\
\Lambda^{2}=72 A_{1} / v^{2} \Omega A_{5}
\end{gathered}
$$

In the calculation, the threshold speed of whirl instability is determined with satisfying Eq.(1) and Eqs.(27) and (28).

\section{E. Nonlinear Transient Stability Analysis}

The nonlinear transient analysis can analyze the journal center locus without the assumption of the small sine vibration to journal behaviors. This analysis requires the solution of equations of motion (Eq.(14) and Eq.(15)) coupled with the lubrication equation (Eq.(1)) as a function of time. The journal center orbits are calculated with repeating two processes, shown as follows.

1) To calculate the fluid film force for the position and the velocity of the journal center at $\tau=\tau_{m}$, solving the lubrication equation.

2) To calculate the position and the velocity of the journal center at $\tau=\tau_{m+1}$, solving the equations of motion by the Runge-Kutta-Gill method.

In this study, it is considered that the bearing system is stable if the journal center orbit converges to each steady-state point while unstable if it diverges.

\section{RESULTS AND DISCUSSION}

Fig. 3 shows the threshold speed of whirl instability for various groove geometries. This figure means that the bearing induces whirl instability at the rotational speed above the threshold line. Fig. 4 shows the relation between the whirl frequency ratio and the groove depth ratio. In these figures, the groove depth $(H)$ and the groove width ratio $(\alpha)$ are parametrically changed. Although the groove angel is one of the groove design parameters, the effect on the threshold speed was small in this analysis. For that reasons, the results for the groove angle of 20 degree are shown in this paper.

The solid lines are the results by the analysis with the consideration of the centrifugal growth whereas the broken lines are those without it. When the bearing specification remains constant, the groove is deeper as $H$ increases. Note that $H=1$ means the results for the plane bearing. The mass of the shaft is heavier as $\Omega$ increases.

As seen in Fig. 3, the threshold speed of whirl instability for $\Omega=0.5$ is greater than that for $\Omega=1.0$. As mentioned later, since the effect of the eccentricity ratio on the threshold speed is not large in this bearing system, the results for $\varepsilon=0.1$ are shown here. Fig. 3 shows the effect of the centrifugal growth of the shaft on the threshold speed. This effect is significant in high dimensionless rotational speed. In this analysis, the threshold speed computed with the consideration of the centrifugal growth is higher than that without it. It is supposed that in the bearing analyzed here, the decrement of the film thickness by the centrifugal growth of the shaft resulted in improving the bearing stability. This study simulated an aluminum shaft which was used in the past bearing test [16]. It can be mentioned that not only to design the herringbone groove parameters but also to control the centrifugal growth of rotating shaft is also important to increase the threshold speed of whirl instability. Fig. 3 makes clear that the bearing with the groove parameters of $0.3<\alpha<0.5$ and $1.5<H<2.0$ have higher threshold speed of whirl instability. Note that considering the centrifugal growth, the proper values of $\alpha$ and $H$ slightly shift to the left in the figure. From Fig. 4, for $H<1.5$ the whirl frequency ratio remains constant of $v=0.5$. On the other hand, for $1.5 \leqq H$ the whirl frequency ratio is changed with the groove parameters. In addition, it increases as the groove width ratio increases.

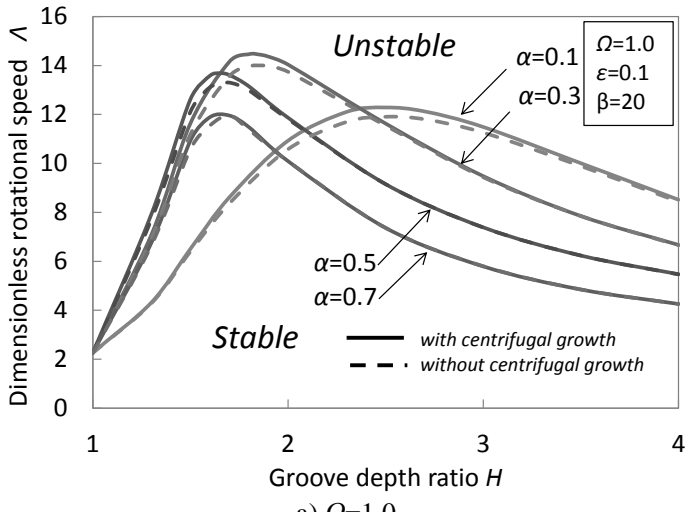

a) $\Omega=1.0$

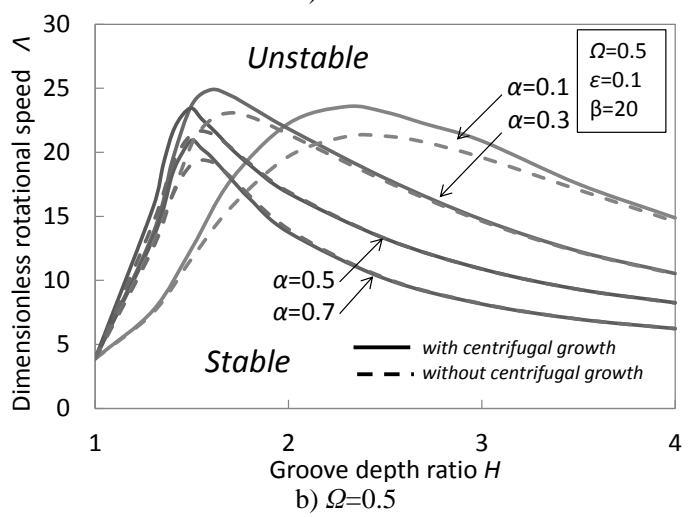

Fig. 3. The threshold speed of whirl instability for various groove geometries.

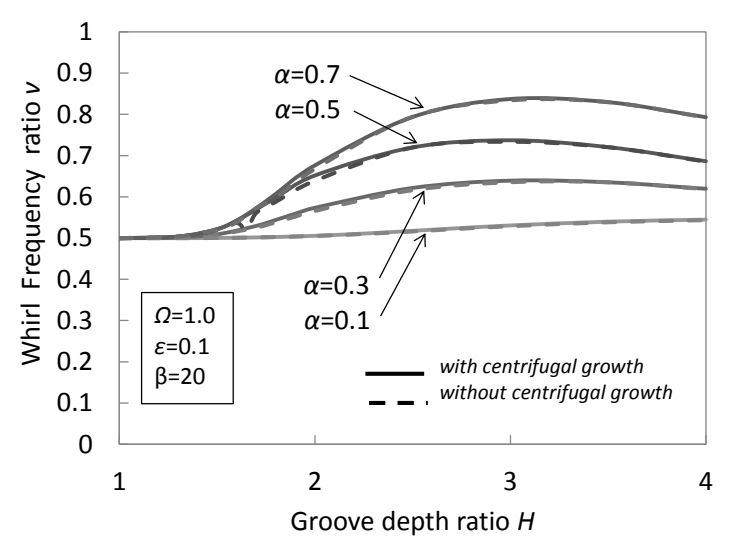

a) $\Omega=1.0$ 


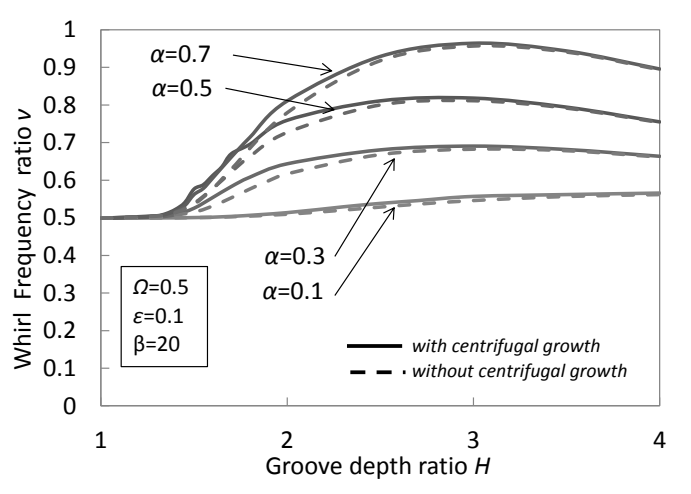

b) $\Omega=0.5$

Fig. 4. Relations between whirl frequency ratio and groove parameters.

Fig. 5 shows the threshold speed of whirl instability for each eccentricity ratio, it is so-called stability map. In this figure, the left side from the stability threshold line is stable whereas the opposite side is unstable. When the shaft is operated on the unstable region in the map, the shaft induces the whirl instability. As seen in Fig. 5, the eccentricity ratio has weak effect on the threshold speed in this bearing. Fig. 6 shows the results of the nonlinear transient analysis at the point $\mathrm{A}, \mathrm{B}$ and $\mathrm{C}$ on the operating curve shown in Fig. 5. When the bearing is stable as represented at the point A, which is left the threshold line, it has a converging orbit. As time progress the shaft settle down to a steady state position. The unstable system as represented at the point $\mathrm{C}$, which is right the threshold line. It has a diverging orbit. As time progress the shaft is going to hit the bushing and it must lead to severe bearing failure. On the threshold line the system represented at the point $\mathrm{B}$ has the limit cycle, in which the orbit neither converges nor diverges with time. These results obtained from the nonlinear transient stability analysis are in good agreement with that from the linear perturbation stability analysis.

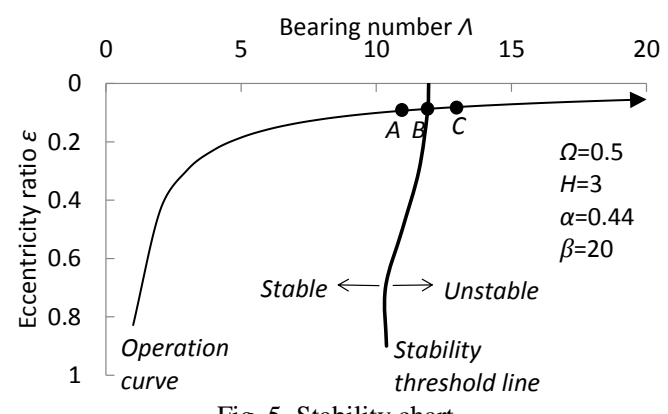

Fig. 5. Stability chart.
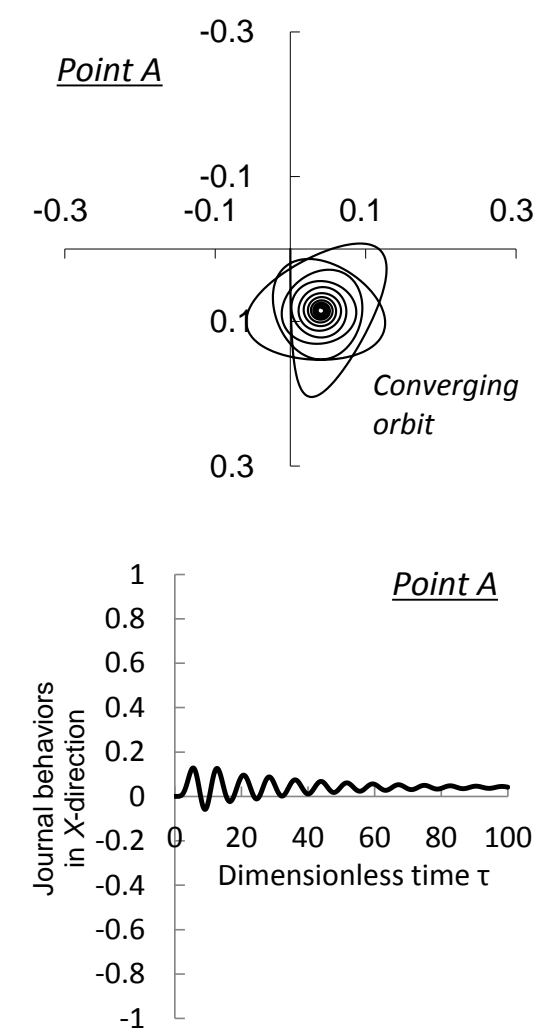

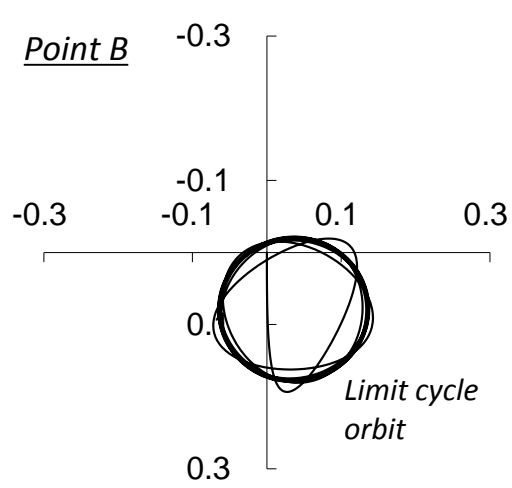

a) Journal center orbits

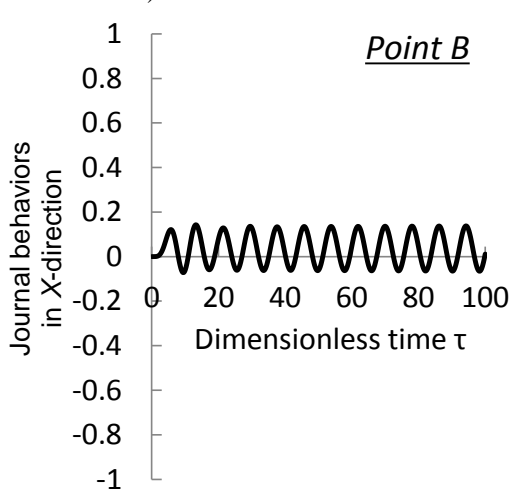

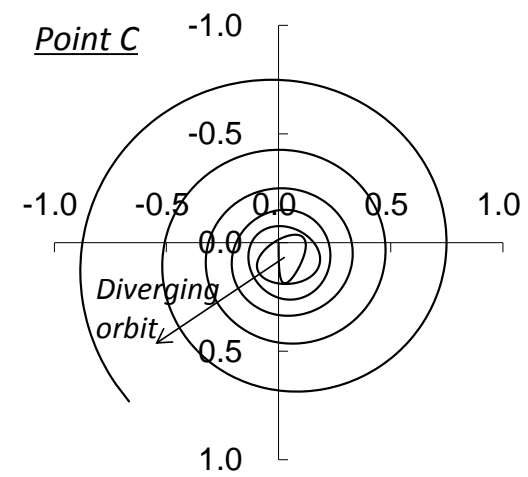

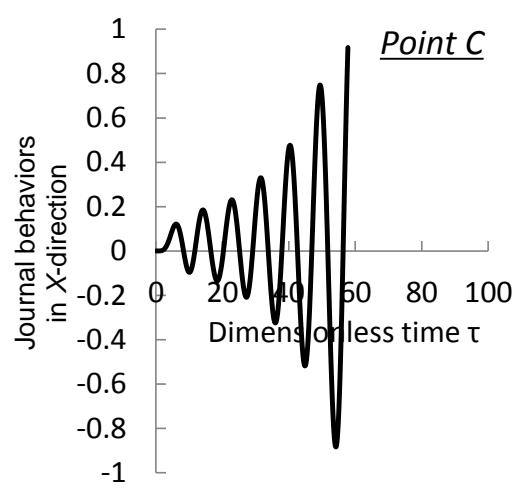

b) Relations between journal behaviors in X-direction and time

Fig. 6. Analytical results by the nonlinear transient analysis for the operating conditions at A, B and C.

\section{A. Example}

When the groove parameters is set to be $\Omega=0.5, H=1.6$, $\alpha=0.3$, and $\beta=20 \mathrm{deg}$, the threshold speed of whirl instability is $\Lambda=25$ as shown in Fig. 3. For this case study, let us consider the following bearing specifications, which was identical to the past bearing test [16].

$$
\begin{gathered}
\hat{C}=4 \times 10^{-6} \mathrm{~m}, \hat{R}=3 \times 10^{-3} \mathrm{~m}, \hat{L}=6 \times 10^{-3} \mathrm{~m}, \\
\hat{\mu}=1.8 \times 10^{-5} \mathrm{~Pa} \cdot \mathrm{s}, \hat{p}_{a}=101325 \mathrm{~Pa}
\end{gathered}
$$

Using these values, the threshold speeds are computed to be about $\hat{N}=0.39 \times 10^{6}$ and $\hat{N}=0.20 \times 10^{6}$ rpm for 2.3 and $4.6 \mathrm{~g}$ weight shaft, respectively.

\section{CONCLusions}

In this study, the stability analysis of the herringbone grooved aerodynamic journal bearings was performed to clarify the proper groove geometry for further ultra 
high-speed operations. The results obtained here are as follows.

1) The effect of the centrifugal growth of rotating shaft was significant in high dimensionless rotational speed.

2) The proper groove geometry for ultrahigh speed operations was $0.3<\alpha<0.5$ and $1.5<H<2.0$. Considering the centrifugal growth of the shaft, the proper values of $\alpha$ and $H$ slightly decreased.

\section{REFERENCES}

[1] J. Powell, "A study of high speed machines with rubber stabilized air bearings," ASME J. Lub. Tech., vol. 90, pp. 701-708, 1968.

[2] G. Belforte, "High-speed rotor with air bearings mounted on flexible supported test bench and experimental results," ASME J. Trib., vol. 130, pp. 021103, 2008.

[3] F. Ehrich and S. Jacobson, "Development of high-speed gas bearings for high-power density microdevices," ASME J. Eng. Gas Turbines Power, vol. 125, pp. 141-148, 2003.

[4] K. Hikichi, K. Shiratori, S. Togo, and K. Hioka, "Hydroinertia gas bearings and their application to high speed micro spinners," J. Jpn. Trib., vol. 50, pp. 465-470, 2005.

[5] S. Tanaka, K. Isomura, S. Togo, and M. Esashi, "Turbo test rig with hydroinertia air bearings for a palmtop gas turbine," J. Micromech. Microeng., vol. 16, pp. 1449-1454, 2005.

[6] S. Tanaka, M. Esashi, K. Isomura, K. Hikichi, Y. Endo, and S. Togo, "Hydroinertia gas bearing system to achieve $470 \mathrm{~m} / \mathrm{s}$ tip speed of 10mm-diameter impellers," ASME J. Trib., vol. 129, pp. 655-659, 2007.

[7] H. Hashmat, "Advancements in the performance of aerodynamic foil journal bearings: High speed and load capability," ASME J. Trib., vol. 116, pp. 287-294, 1994.

[8] M. Salehi, H. Heshmat, J. Waltonm, and M. Tomaszewski, "Operation of a mesoscopic gas turbine simulator at speeds in excess of $700,000 \mathrm{rpm}$ on foil bearings," ASME J. Eng. Gas Turbines Power, vol. 126, pp. 170-176, 2004.

[9] D. Kim, "Mesoscale foil gas bearings for palm-sized turbomachinery," ASME J. Engineering for Gas Turbines Power, vol. 131, 2009.

[10] D. Hong, B. Woo, J. Lee, and D. Koo, "Ultra high speed motor supported by air foil bearings for air lower cooling fuel cells," IEEE Transactions, vol. 48, pp. 871-874, 2012

[11] G. Jang and J. Yoon, "Stability analysis of a hydrodynamic journal bearing with rotating herringbone grooves," ASME J. Trib., vol. 125, pp. 291-300, 2003

[12] S. Yoshimoto and A. Takahashi, "A method of reducing windage power loss in a laser scanner mirror by using the pumping effect of herrinebone-grooved gas journal bearings," ASME J. Trib., vol. 121, pp. 506-509, 1999 .

[13] D. Bonneau, "Analysis of aerodynamic journal bearings with small number of herringbone grooves by finite element method," ASME $J$. Trib., vol. 116, pp. 698-704, 1994.

[14] T. Kobayashi, "Numerical analysis of herringbone-grooved gas-lubricated journal bearings using multigrid technique," ASME J. Trib., vol. 121, pp. 148-156, 1999.

[15] M. Sahu, "Thermo-hydrodynamic analysis of herringbone grooved journal bearings," Trib. Int., vol. 36, pp. 1395-1404, 2006.

[16] N. Miyanaga and J. Tomioka, "Development of herringbone-grooved aerodynamic journal bearing systems for ultra-high-speed rotations," Key Engineering Materials.

[17] S. Yoshimoto and K. Yagi, "Development of arodynamic bearings for an ultra-micro gas turbine," in Proc. JSME Annual Meeting, 2003, pp. 281-282.

[18] T. Ise, S. Onoe, T. Asami, I. Kawashima, and T. Maeda, "Split-type herringbone-grooved hydrodynamic journal gas bearings fro small ultra-high-speed rotary machines," J. Power and Energy Systems, vol. 5, pp. 406-413, 2011.

[19] J. Vorh, "Characteristics of herringbone grooved gas-lubricated journal bearings," ASME, J. Basic Engineering, vol. 87, pp. 568-578, 1965.

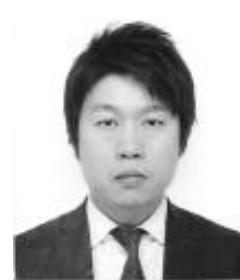

Norifumi Miyanaga was bone in Fukui, Japan, in 1980. He attended the Graduate School of Science and Engineering, Waseda University, and earned his doctor of engineering degree in 2012.

$\mathrm{He}$ is an assistant professor of the Department of Science and Engineering, Kanto-Gakuin University, Japan. His major research field is tribology, especially in fluid film lubrication.

Assistant Prof. Norifumi Miyanaga is a member of Japan Society for Design Engineering (JSDE), Japanese Society of Tribologists (JAST), the Japan Scociety of Mechanical Engineers (JSME).

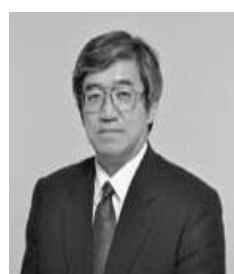

lubrication.

Prof. Jun Tomioka is a member of the Japan Society of Mechanical Engineers (JSME). He is currently the vice president of Japanese Society of Tribologists (JAST), and also the vice president of Japan Society for Design Engineering (JSDE).

Jun Tomioka was born in Kyoto, Japan, in 1959. He graduated from the Graduate School of Science and Engineering, Waseda University in $1989 . \mathrm{He}$ received the doctor of engineering from Waseda University in 1989.

$\mathrm{He}$ is a professor of the Faculty of Science and Engineering, Waseda Unviersity, Japan. His major research field is tribology, especially in fluid film

(1)

